\title{
A QUENCHED CENTRAL LIMIT THEOREM FOR REVERSIBLE RANDOM WALKS IN A RANDOM ENVIRONMENT ON $\mathbb{Z}$
}

\author{
HOANG-CHUONG LAM,* Can Tho University and Ben Gurion University
}

\begin{abstract}
The main aim of this paper is to prove the quenched central limit theorem for reversible random walks in a stationary random environment on $\mathbb{Z}$ without having the integrability condition on the conductance and without using any martingale. The method shown here is particularly simple and was introduced by Depauw and Derrien [3]. More precisely, for a given realization $\omega$ of the environment, we consider the Poisson equation $\left(P_{\omega}-I\right) g=f$, and then use the pointwise ergodic theorem in [8] to treat the limit of solutions and then the central limit theorem will be established by the convergence of moments. In particular, there is an analogue to a Markov process with discrete space and the diffusion in a stationary random environment.
\end{abstract}

Keywords: Quenched central limit theorem; reversible random walk in random environment

2010 Mathematics Subject Classification: Primary 60J15; 60F05

Secondary 60J27; $60 \mathrm{~J} 60$

\section{Introduction}

Let $(\Omega, \mathcal{A}, \mathbb{P})$ be a probability space. The definition of a random walk in a random environment involves two ingredients:

1. The environment which is randomly chosen but remains fixed throughout the time evolution.

2. The random walk whose transition probability is determined by the environment.

The space $\Omega$ is interpreted as the space of environments. For each $\omega \in \Omega$, we define the random walk in the environment $\omega$ as the (time homogeneous) Markov chain $\left(X_{n}\right)_{n \geq 0}$ on $\mathbb{Z}$ with certain (random) transition probabilities

$$
p(\omega, x, y)=\mathbb{P}_{\omega}\left\{X_{1}=y / X_{0}=x\right\} .
$$

The probability measure $\mathbb{P}_{\omega}$ determines the distribution of the random walk in a given environment $\omega$. In this paper we study only the random walk with the initial condition $X_{0}=0$,

$$
\mathbb{P}_{\omega}^{0}\left\{X_{0}=0\right\}=1 .
$$

The probability measure $\mathbb{P}_{\omega}^{0}$ indicates the distribution of the random walk in a given environment $\omega$ with the initial position of the walk referred to as the Quenched law. For more information about the random walk in a random environment and the Annealed law see [1], [6], and [9].

Received 8 May 2013; revision received 13 January 2014.

* Postal address: Department of Mathematics, Can Tho University, Can Tho City, Vietnam.

Email address: lhchuong@ctu.edu.vn 
Now we consider the following model for the random walk in a random environment. Let $(\Omega, \mathcal{A}, \mu)$ be a probability space and $T$ be an invertible measure preserving transformation on $\Omega$, which is ergodic. More precisely, $T$ acts on $\Omega$ by

$$
T: \Omega \times \mathbb{Z} \rightarrow \Omega \text { and }(\omega, k) \mapsto T^{k} \omega,
$$

which is joint measurable and satisfies

1. For any $k, h \in \mathbb{Z}: T^{k+h}=T^{k} T^{h}$ and $T^{0} \omega=\omega$.

2. $T$ preserves the measure $\mu: \mu\left(T^{k} A\right)=\mu(A)$ for any $k \in \mathbb{Z}$.

3. $T$ is ergodic: if $T^{k} A=A$ (up to null sets) for all $k \in \mathbb{Z}$ then $\mu(A)$ is equal to 0 or 1 .

On the $\mathbb{Z}$ network, we assume that the conductivity of the edge between $\{k, k+1\}$ is equal to $c\left(T^{k} \omega\right)$, where $c$ is a positive measurable function on $\Omega$. Fix $\omega \in \Omega$ and consider a random walk $\left(X_{n}\right)_{n \geq 0}$ on $\mathbb{Z}$ where $X_{0}=0$ and the transition probability $p(\omega, k, h)$ is given by

$$
p(\omega ; k, k+1)=\mathbb{P}_{\omega}^{0}\left\{X_{n+1}=k+1 / X_{n}=k\right\}=c\left(T^{k} \omega\right) / \bar{c}\left(T^{k} \omega\right),
$$

and

$$
p(\omega ; k, k-1)=\mathbb{P}_{\omega}^{0}\left\{X_{n+1}=k-1 / X_{n}=k\right\}=c\left(T^{k-1} \omega\right) / \bar{c}\left(T^{k} \omega\right),
$$

where $\bar{c}(\omega)=c(\omega)+c\left(T^{-1} \omega\right)$. These random walks are reversible since for all adjacent vertices $x, y$ in $\mathbb{Z}$, we have $\bar{c}\left(T^{x} \omega\right) p(\omega ; x, y)=\bar{c}\left(T^{y} \omega\right) p(\omega ; y, x)$. The Markov operator $f \mapsto P_{\omega} f$ is defined by

$$
P_{\omega} f(k)=\frac{1}{\bar{c}\left(T^{k} \omega\right)}\left[c\left(T^{k-1} \omega\right) f(k-1)+c\left(T^{k} \omega\right) f(k+1)\right] .
$$

When $c$ is integrable but $c^{-1}$ not, Derriennic and Lin have proved, in an unpublished work, the annealed central limit theorem (CLT) with null variance: $\lim _{n \rightarrow \infty} n^{-1} \mathbb{E}_{\omega}\left(X_{n}^{2}\right)=0$ in $\mu$ measure, where $\mathbb{E}_{\omega}$ denotes the expectation relative to the randomness of the walk with the environment being fixed. For the quenched version Depauw and Derrien [3] considered a nonnegative solution $f$, defined on $\mathbb{Z}$, of the Poisson equation $\left(P_{\omega}-I\right) f=1$ and that satisfies $f(0)=0$ to obtain the limit of the variance of the reversible random walk $\left(X_{n}\right)_{n \geq 0}$ without using any martingale and without having any condition on function $c$ except that $c>0$.

Theorem 1. (Depauw and Derrien [3].) For almost all environments $\omega$,

$$
\lim _{n \rightarrow+\infty} \mathbb{E}_{\omega}\left\{\frac{X_{n}^{2}}{n}\right\}=\left[\int \frac{1}{c} \mathrm{~d} \mu \int c \mathrm{~d} \mu\right]^{-1} .
$$

This limit is null if at least one of the integrals is $+\infty$.

When both of $c$ and $c^{-1}$ are integrable, the quenched CLT, in the usual case, can be proved by the method of martingale, first introduced by Kozlov, [5]. Unfortunately, this method does not allow us to treat the case when $c$ or $c^{-1}$ are not integrable. The aim of this paper is to generalize Theorem 1 and to establish the quenched CLT without using any martingale and without having any condition on function $c$ except that $c>0$. In the case when at least one of $c$ and $c^{-1}$ is not integrable, $X_{n} / \sqrt{n}$ converges to the degenerate normal distribution. The second method is adapted from [3] and leads to the following theorem. 
Theorem 2. For almost all environments $\omega$,

$$
\frac{X_{n}}{\sqrt{n}} \stackrel{\mathrm{D}}{\rightarrow} \mathcal{N}\left(0,\left[\int \frac{1}{c} \mathrm{~d} \mu \int c \mathrm{~d} \mu\right]^{-1}\right) \text { as } n \rightarrow+\infty .
$$

The limiting distribution being a degenerate normal distribution if at least one of the integrals is $+\infty$.

Throughout this paper, $\stackrel{\text { D }}{\rightarrow}$ ' denotes the convergence in distribution and $\mathcal{N}\left(\lambda, \sigma^{2}\right)$ denotes the normal distribution with mean $\lambda$ and variance $\sigma^{2}$.

This paper is organized as follows. In Section 2 we prove Theorem 2. In Section 3 there is an analogue to a Markov process with continuous time and discrete space. Finally, in Section 4 we consider the diffusion in a random environment. It is somewhat involved but we will show the proof of the CLT explicitly.

\section{Proof of Theorem 2}

Consider a normal distribution $Z \sim \mathcal{N}\left(0, \sigma^{2}\right)$, and for each $\ell=1,2,3, \ldots$, we have

$$
\mathbb{E}\left\{Z^{\ell}\right\}=0 \quad \text { if } \ell=2 k-1 \quad \text { and } \quad \mathbb{E}\left\{Z^{\ell}\right\}=\frac{(2 k) !}{k ! 2^{k}} \sigma^{\ell} \quad \text { if } \ell=2 k .
$$

Using the method of moments introduced in [2, Theorem 30.2, page 390] to prove Theorem 2 it is sufficient to show that for almost all environments $\omega$

$$
\lim _{n \rightarrow+\infty} \mathbb{E}_{\omega}\left\{\left(\frac{X_{n}}{\sqrt{n}}\right)^{\ell}\right\}=\mathbb{E}\left\{Z^{\ell}\right\}= \begin{cases}0 & \text { if } \ell=2 k-1 \\ \frac{(2 k) !}{k ! 2^{k}} \sigma^{\ell} & \text { if } \ell=2 k\end{cases}
$$

for each $\ell=1,2,3, \ldots$ In this case $\sigma^{2}=\left[\int(1 / c) \mathrm{d} \mu \int c \mathrm{~d} \mu\right]^{-1}$.

We begin with the following elementary lemma.

Lemma 1. Let $u_{n}$, $v_{n}$ be two sequences of positive real numbers and a nonnegative integer $\alpha \in \mathbb{N}$. Assume that $\lim _{n \rightarrow \infty}(1 / n) \sum_{\ell=1}^{n} u_{\ell}=u>0$ and $\lim _{n \rightarrow \infty} v_{n}=v>0$. If both of $u$ and $v$ are finite

$$
\lim _{n \rightarrow \infty} \frac{1}{n^{\alpha+1}} \sum_{\ell=1}^{n} \ell^{\alpha} u_{\ell} v_{\ell}=\frac{u v}{\alpha+1}
$$

else if at least one of $u$ and $v$ is infinite then the limit in (1) being $+\infty$.

Proof. We will prove that both of $u$ and $v$ are finite, with the other cases left to the reader. For $\alpha=0$, we will show that

$$
\lim _{n \rightarrow \infty} \frac{1}{n} \sum_{\ell=1}^{n} u_{\ell} v_{\ell}=u v
$$

We have

$$
\begin{aligned}
\left|\frac{1}{n} \sum_{\ell=1}^{n} u_{\ell} v_{\ell}-u v\right| & \leq\left|\frac{1}{n} \sum_{\ell=1}^{n} u_{\ell}\left(v_{\ell}-v\right)\right|+\left|\frac{1}{n} \sum_{\ell=1}^{n}\left(u_{\ell}-u\right) v\right| \\
& \leq \frac{1}{n} \sum_{\ell=1}^{n} u_{\ell}\left|v_{\ell}-v\right|+v\left|\frac{1}{n} \sum_{\ell=1}^{n} u_{\ell}-u\right| \\
& <\varepsilon
\end{aligned}
$$

for any $\varepsilon>0$ when $n$ large enough, completing the proof of (2). 
Now assume that (1) is true for $\alpha \geq 0$. We claim that it also holds for $\alpha+1$, that is

$$
\lim _{n \rightarrow \infty} \frac{1}{n^{\alpha+2}} \sum_{\ell=1}^{n} \ell^{\alpha+1} u_{\ell} v_{\ell}=\frac{u v}{\alpha+2}
$$

Let $W_{n}=\sum_{\ell=1}^{n} \ell^{\alpha} u_{\ell} v_{\ell}$, using Abel's transformation

$$
\frac{1}{n^{\alpha+2}} \sum_{\ell=1}^{n} \ell^{\alpha+1} u_{\ell} v_{\ell}=-\frac{1}{n^{\alpha+2}} \sum_{\ell=1}^{n-1} W_{\ell}+\frac{1}{n^{\alpha+1}} W_{n}=-I_{1}+I_{2}
$$

By the assumption $\lim _{n \rightarrow \infty} I_{2}=\lim _{n \rightarrow \infty}\left(1 / n^{\alpha+1}\right) W_{n}=u v /(\alpha+1)$, we have

$$
\begin{aligned}
&\left|I_{1}-\frac{u v}{(\alpha+1)(\alpha+2)}\right| \leq \frac{1}{n^{\alpha+2}} \sum_{\ell=1}^{n-1} \ell^{\alpha+1}\left|\frac{W_{\ell}}{\ell^{\alpha+1}}-\frac{u v}{\alpha+1}\right| \\
&+\left|\frac{1}{n^{\alpha+2}} \sum_{\ell=1}^{n-1} \ell^{\alpha+1}-\frac{1}{\alpha+2}\right| \frac{u v}{\alpha+1} \\
&<\varepsilon
\end{aligned}
$$

for any $\varepsilon>0$ when $n$ is large enough since $\left(1 / n^{\alpha+2}\right) \sum_{\ell=1}^{n-1} \ell^{\alpha+1}=(1 / n) \sum_{\ell=1}^{n-1}(\ell / n)^{\alpha+1}$ and as $n$ goes to $\infty$ we will have the limit that is equal to $\int_{0}^{l} x^{\alpha+1} \mathrm{~d} x=1 /(\alpha+2)$. It follows that $\lim _{n \rightarrow \infty} I_{1}=u v /((\alpha+1)(\alpha+2))$. Hence,

$$
\lim _{n \rightarrow \infty} \frac{1}{n^{\alpha+2}} \sum_{\ell=1}^{n} \ell^{\alpha+1} u_{\ell} v_{\ell}=-\frac{u v}{(\alpha+1)(\alpha+2)}+\frac{u v}{\alpha+1}=\frac{u v}{\alpha+2},
$$

which completes the proof of (3).

Lemma 2. Given a function $\psi: \mathbb{Z} \rightarrow \mathbb{R}$ there exists a unique function $\phi: \mathbb{Z} \rightarrow \mathbb{R}$ such that

$$
\left(P_{\omega}-I\right) \phi=\psi, \quad \phi(1)=a, \quad \phi(-1)=b .
$$

Proof. Since

$$
\left(P_{\omega}-I\right) \phi(0)=\psi(0)
$$

we have

$$
c\left(T^{-1} \omega\right) \phi(-1)+c(\omega) \phi(1)-\bar{c}(\omega) \phi(0)=\bar{c}(\omega) \psi(0) .
$$

This determines $\phi(0)$.

For $m \geq 2$, we consider

$$
\left(P_{\omega}-I\right) \phi(m-1)=\psi(m-1)
$$

This is equivalent to

$$
c\left(T^{m-1} \omega\right)[\phi(m)-\phi(m-1)]=c\left(T^{m-2} \omega\right)[\phi(m-1)-\phi(m-2)]+\bar{c}\left(T^{m-1} \omega\right) \psi(m-1)
$$

and then by induction on $m$

$$
\phi(m)=\phi(1)+[\phi(1)-\phi(0)] \sum_{\ell=1}^{m-1} \frac{c(\omega)}{c\left(T^{\ell} \omega\right)}+\sum_{\ell=1}^{m-1} \frac{1}{c\left(T^{\ell} \omega\right)} \sum_{s=1}^{\ell} \bar{c}\left(T^{s} \omega\right) \psi(s) .
$$


Similarly for $m \leq-2$, we have

$$
\phi(m)=\phi(-1)+[\phi(-1)-\phi(0)] \sum_{\ell=2}^{-m} \frac{c\left(T^{-1} \omega\right)}{c\left(T^{-\ell} \omega\right)}+\sum_{\ell=2}^{-m} \frac{1}{c\left(T^{-\ell} \omega\right)} \sum_{s=1}^{\ell-1} \bar{c}\left(T^{-s} \omega\right) \psi(-s) .
$$

We have thus proved that $\phi$ is a unique solution of (4). We also deduce that a particular solution $\phi$ of the Poisson equation $\left(P_{\omega}-I\right) \phi=\psi$ is characterized by the values $\phi(-1), \phi(0)$ and $\phi(1)$ such that they satisfy (5).

Proposition 1. For almost all environments $\omega$, we have

$$
\lim _{n \rightarrow+\infty} \mathbb{E}_{\omega}\left\{\left(\frac{X_{n}}{\sqrt{n}}\right)^{2 k}\right\}=\frac{(2 k) !}{2^{k} k !} \sigma^{2 k}
$$

for each $k \geq 1$. This limit is null if at least one of $c$ and $c^{-1}$ is not integrable.

This is the generalization of Theorem 1 (or [3, Theorem 0.1]).

Proof. Fixing $\omega \in \Omega$ we consider a sequence of functions $f_{k} \geq 0$, defined on $\mathbb{Z}$, such that

$$
\left(P_{\omega}-I\right) f_{k} \equiv f_{k-1} \quad \text { for } k \geq 1, \quad f_{0} \equiv 1, \quad f_{k}(0)=0 \quad \text { for } k \geq 1 .
$$

By Lemma 2 we can determine the function $f_{1}$ which satisfies $f_{1}(-1)=\bar{c}(\omega) / c\left(T^{-1} \omega\right)$ and $f_{1}(1)=0$

$$
f_{1}(m)= \begin{cases}\sum_{\ell=1}^{m-1} \frac{1}{c\left(T^{\ell} \omega\right)} \sum_{s=1}^{\ell} \bar{c}\left(T^{s} \omega\right) & \text { if } m \geq 2, \\ 0 & \text { if } m=0,1, \\ \sum_{\ell=1}^{-m} \frac{1}{c\left(T^{-\ell} \omega\right)} \sum_{s=0}^{\ell-1} \bar{c}\left(T^{-s} \omega\right) & \text { if } m \leq-1\end{cases}
$$

and for $k \geq 2$ the function $f_{k}$ which satisfies $f_{k}(1)=f_{k}(-1)=0$

$$
f_{k}(m)= \begin{cases}\sum_{\ell=1}^{m-1} \frac{1}{c\left(T^{\ell} \omega\right)} \sum_{s=1}^{\ell} \bar{c}\left(T^{s} \omega\right) f_{k-1}(s) & \text { if } m \geq 2, \\ 0 & \text { if } m=-1,0,1, \\ \sum_{\ell=2}^{-m} \frac{1}{c\left(T^{-\ell} \omega\right)} \sum_{s=1}^{\ell-1} \bar{c}\left(T^{-s} \omega\right) f_{k-1}(-s) & \text { if } m \leq-2 .\end{cases}
$$

Then, for any integer $m$ and for $k \geq 1$, we have

$$
\left(P_{\omega}-I\right) f_{k}(m)=f_{k-1}(m) .
$$

Replace $m$ by $X_{n}$ and take the expectation to obtain

$$
\mathbb{E}_{\omega}\left\{f_{k}\left(X_{n+1}\right)\right\}=\mathbb{E}_{\omega}\left\{f_{k}\left(X_{n}\right)\right\}+\mathbb{E}_{\omega}\left\{f_{k-1}\left(X_{n}\right)\right\}
$$

for any $n \geq 0$. It follows that for each $k \geq 1$

$$
\mathbb{E}_{\omega}\left\{f_{k}\left(X_{n}\right)\right\} \sim \frac{n^{k}}{k !}
$$

when $n$ is large enough since $f_{k}(0)=0$ by the definition of $f_{k}$ and $X_{0}=0$ by the assumption of the random walk $X_{n}$. The proof of (6) is by induction on $k$. 
Equation (6) can be rewritten as

$$
\mathbb{E}_{\omega}\left\{\frac{f_{k}\left(X_{n}\right)}{X_{n}^{2 k}} \frac{X_{n}^{2 k}}{n^{k}}\right\} \sim \frac{1}{k !} .
$$

We see that $\lim _{m \rightarrow \infty} f_{k}(m) / m^{2 k}$ exists and so $\lim _{n \rightarrow+\infty} \mathbb{E}_{\omega}\left\{X_{n}^{2 k} / n^{k}\right\}$ exists, completing the proof.

In the next step we will compute the limit of $f_{k}(m) / m^{2 k}$ by using the pointwise ergodic theorem (p.e.t.) and Lemma 1.

Lemma 3. For each $k \geq 1$, with $f_{k}$ defined as above, we have

$$
\lim _{m \rightarrow \pm \infty} \frac{f_{k}(m)}{m^{2 k}}=\frac{2^{k}}{(2 k) !} \sigma^{-2 k}
$$

This limit is $+\infty$ if at least one of $c$ and $c^{-1}$ is not integrable. It is denoted by $c_{k}$.

Proof. This limit is true for $k=1$. Indeed, for the case $m>0$, we have

$$
\frac{f_{1}(m)}{m^{2}}=\frac{1}{m} \sum_{\ell=1}^{m-1}\left(\frac{\ell}{m}\right) \frac{1}{c\left(T^{\ell} \omega\right)} \frac{1}{\ell} \sum_{s=1}^{\ell} \bar{c}\left(T^{s} \omega\right) .
$$

Applying Lemma 1 and the p.e.t. for $u_{\ell}=1 / c\left(T^{\ell} \omega\right), v_{\ell}=(1 / \ell) \sum_{s=1}^{\ell} \bar{c}\left(T^{s} \omega\right)$ and $\alpha=1$ this tends to $\sigma^{-2}=\left[\int(1 / c) \mathrm{d} \mu \int c \mathrm{~d} \mu\right]$. The point is that, since $c>0$, the convergence is still satisfied if one of these integrals is $+\infty$ (see [3]).

Assume that (7) is also true for $k \geq 1$, we claim that it holds for $k+1$, that is

$$
\lim _{m \rightarrow \infty} \frac{f_{k+1}(m)}{m^{2(k+1)}}=\frac{2^{k+1}}{(2 k+2) !} \sigma^{-2(k+1)} .
$$

We have

$$
\frac{1}{\ell^{2 k+1}} \sum_{s=1}^{\ell} \bar{c}\left(T^{s} \omega\right) f_{k}(s)=\frac{1}{\ell} \sum_{s=1}^{\ell}\left(\frac{s}{\ell}\right)^{2 k} \bar{c}\left(T^{s} \omega\right) \frac{1}{s^{2 k}} f_{k}(s) .
$$

Again, applying Lemma 1 and the p.e.t. for $u_{s}=\bar{c}\left(T^{s} \omega\right), v_{s}=\left(1 / s^{2 k}\right) f_{k}(s)$ and $\alpha=2 k$, this tends to $\int_{\Omega} c \mathrm{~d} \mu 2^{k+1} /(2 k+1) ! \sigma^{-2 k}$. Moreover,

$$
\frac{f_{k+1}(m)}{m^{2(k+1)}}=\frac{1}{m} \sum_{\ell=1}^{m-1}\left(\frac{\ell}{m}\right)^{2 k+1} \frac{1}{c\left(T^{\ell} \omega\right)} \frac{1}{\ell^{2 k+1}} \sum_{s=1}^{\ell} \bar{c}\left(T^{s} \omega\right) f_{k}(s)
$$

Again, applying Lemma 1 and the p.e.t. for $u_{\ell}^{\prime}=1 / c\left(T^{\ell} \omega\right), v_{\ell}^{\prime}=\left(1 / \ell^{2 k+1}\right) \sum_{s=1}^{\ell} \bar{c}\left(T^{s} \omega\right) f_{k}(s)$ and $\alpha=2 k+1$, this tends to $2^{k+1} /(2(k+1)) ! \sigma^{-2(k+1)}$ which completes the proof of (8).

Similarly, we have the same result for the case $m<0$.

From Lemma 3, for any $\varepsilon>0$, there exists $M>0$ such that for any $m>M$

$$
\left|\frac{m^{2 k}}{f_{k}(m)}-\frac{1}{c_{k}}\right|<\varepsilon / 2 .
$$


Decomposing $\Omega=\left\{\left|X_{n}\right| \leq M\right\} \cup\left\{\left|X_{n}\right|>M\right\}$ and combining (6) and (9) we obtain

$$
\begin{aligned}
&\left|\mathbb{E}_{\omega}\left\{\left(\frac{X_{n}}{\sqrt{n}}\right)^{2 k}\right\}-\frac{1}{k ! c_{k}}\right| \approx \mid \mathbb{E}_{\omega}\left\{\left(\frac{X_{n}^{2 k}}{f_{k}\left(X_{n}\right)}-\frac{1}{c_{k}}\right) \frac{f_{k}\left(X_{n}\right)}{n^{k}} \mathbf{1}_{\left\{\left|X_{n}\right|>M\right\}}\right\} \\
&+\frac{1}{n^{k}} \mathbb{E}_{\omega}\left\{\left(X_{n}^{2 k}-\frac{1}{c_{k}} f_{k}\left(X_{n}\right)\right) \mathbf{1}_{\left\{\left|X_{n}\right| \leq M\right\}}\right\} \mid \\
& \leq \mathbb{E}_{\omega}\left\{\left|\frac{X_{n}^{2 k}}{f_{k}\left(X_{n}\right)}-\frac{1}{c_{k}}\right| \frac{f_{k}\left(X_{n}\right)}{n^{k}} \mathbf{1}_{\left\{\left|X_{n}\right|>M\right\}}\right\} \\
&+\frac{1}{n^{k}} \mathbb{E}_{\omega}\left\{\left|X_{n}^{2 k}-\frac{1}{c_{k}} f_{k}\left(X_{n}\right)\right| \mathbf{1}_{\left\{\left|X_{n}\right| \leq M\right\}}\right\} \\
&<\varepsilon
\end{aligned}
$$

for $n$ large enough. Since $\varepsilon$ is as small as we need, we obtain the desired result, which completes Proposition 1.

Proposition 2. For almost all environments $\omega$, it holds that

$$
\lim _{n \rightarrow+\infty} \mathbb{E}_{\omega}\left\{\left(\frac{X_{n}}{\sqrt{n}}\right)^{2 k-1}\right\}=0 \text { for each } k \geq 1 .
$$

Proof. Fixing $\omega \in \Omega$ we consider a sequence of functions $g_{k}$, defined on $\mathbb{Z}$, such that

$$
\left(P_{\omega}-I\right) g_{k} \equiv g_{k-1} \quad \text { for } k \geq 1, \quad g_{0} \equiv 0, \quad g_{k}(0)=0 \quad \text { for } k \geq 1 .
$$

Again, by Lemma 2 we can determine the function $g_{1}$ which satisfies $g_{1}(1)=1 / c(\omega)$ and $g_{1}(-1)=-1 / c\left(T^{-1} \omega\right)$

$$
g_{1}(m)= \begin{cases}\sum_{\ell=0}^{m-1} \frac{1}{c\left(T^{\ell} \omega\right)} & \text { if } m \geq 1 \\ 0 & \text { if } m=0 \\ -\sum_{\ell=1}^{-m} \frac{1}{c\left(T^{-\ell} \omega\right)} & \text { if } m \leq-1\end{cases}
$$

and for $k \geq 2$ the function $g_{k}$ satisfies $g_{k}(1)=g_{k}(-1)=0$

$$
g_{k}(m)= \begin{cases}\sum_{\ell=1}^{m-1} \frac{1}{c\left(T^{\ell} \omega\right)} \sum_{s=1}^{\ell} \bar{c}\left(T^{s} \omega\right) g_{k-1}(s) & \text { if } m \geq 2, \\ 0 & \text { if } m=-1,0,1, \\ \sum_{\ell=2}^{-m} \frac{1}{c\left(T^{-\ell} \omega\right)} \sum_{s=1}^{\ell-1} \bar{c}\left(T^{-s} \omega\right) g_{k-1}(-s) & \text { if } m \leq-2 .\end{cases}
$$

Then for any integer $m$ and for $k \geq 1$

$$
\left(P_{\omega}-I\right) g_{k}(m)=g_{k-1}(m) .
$$

Replace $m$ by $X_{n}$ and then take the expectation to obtain

$$
\mathbb{E}_{\omega}\left\{g_{k}\left(X_{n+1}\right)\right\}=\mathbb{E}_{\omega}\left\{g_{k}\left(X_{n}\right)\right\}+\mathbb{E}_{\omega}\left\{g_{k-1}\left(X_{n}\right)\right\} \text { for any } n \geq 0 .
$$


It is straightforward to see that for each $k \geq 1$

$$
\mathbb{E}_{\omega}\left\{g_{k}\left(X_{n}\right)\right\}=0 \text { for any } n \geq 0 .
$$

Equation (10) can be rewritten as

$$
\mathbb{E}_{\omega}\left\{\frac{g_{k}\left(X_{n}\right)}{X_{n}^{2 k-1}} \times \frac{X_{n}^{2 k-1}}{(\sqrt{n})^{2 k-1}}\right\}=0 .
$$

We see that the limit of $g_{k}(m) / m^{2 k-1}$ exists and so the limit of $\mathbb{E}_{\omega}\left\{\left(X_{n} / \sqrt{n}\right)^{2 k-1}\right\}$ equals 0 .

In the next step we will compute the limit of $g_{k}(m) / m^{2 k-1}$ by using the pointwise ergodic theorem and Lemma 1.

Lemma 4. For each $k \geq 1$ and $g_{k}$ defined as above, we have

$$
\lim _{m \rightarrow \pm \infty} \frac{g_{k}(m)}{m^{2 k-1}}=\frac{2^{k-1}}{(2 k-1) !} \sigma^{-2 k+2} \int_{\Omega} \frac{1}{c} \mathrm{~d} \mu .
$$

This limit is $+\infty$ if at least one of $c$ and $c^{-1}$ is not integrable. It is denoted by $d_{k}$.

The proof of Lemma 4 is left to the reader. From Lemma 4 , for any $\varepsilon>0$, there exists $M>0$ such that for any $|m|>M$

$$
\left|\frac{g_{k}(m)}{m^{2 k-1} d_{k}}-1\right|<\varepsilon .
$$

Decomposing $\Omega=\left\{\left|X_{n}\right| \leq M\right\} \cup\left\{\left|X_{n}\right|>M\right\}$ and combining (10) and (11), we have

$$
\begin{aligned}
\left|\mathbb{E}_{\omega}\left\{\left(\frac{X_{n}}{\sqrt{n}}\right)^{2 k-1}\right\}\right|= & \mid \mathbb{E}_{\omega}\left\{\frac{1}{(\sqrt{n})^{2 k-1}}\left(X_{n}^{2 k-1}-\frac{g_{k}\left(X_{n}\right)}{d_{k}}\right) \mathbf{1}_{\left\{\left|X_{n}\right| \leq M\right\}}\right\} \\
& +\mathbb{E}_{\omega}\left\{\frac{1}{(\sqrt{n})^{2 k-1}}\left(X_{n}^{2 k-1}-\frac{g_{k}\left(X_{n}\right)}{d_{k}}\right) \mathbf{1}_{\left\{\left|X_{n}\right|>M\right\}}\right\} \mid \\
\leq & \mathbb{E}_{\omega}\left\{\frac{1}{\left.(\sqrt{n})^{2 k-1}\left|X_{n}^{2 k-1}-\frac{g_{k}\left(X_{n}\right)}{d_{k}}\right| \mathbf{1}_{\left\{\left|X_{n}\right| \leq M\right\}}\right\}}\right. \\
& +\mathbb{E}_{\omega}\left\{\left(\frac{\left|X_{n}\right|}{\sqrt{n}}\right)^{2 k-1}\left|1-\frac{g_{k}\left(X_{n}\right)}{X_{n}^{2 k-1} d_{k}}\right| \mathbf{1}_{\left\{\left|X_{n}\right|>M\right\}}\right\} \\
\leq & \varepsilon+\varepsilon \sqrt{\mathbb{E}_{\omega}\left\{\left(\frac{X_{n}}{\sqrt{n}}\right)^{2(2 k-1)}\right\}}
\end{aligned}
$$

for $n$ large enough. By Proposition 1 the $\operatorname{limit}_{\lim } \rightarrow \infty \mathbb{E}_{\omega}\left\{\left(X_{n} / \sqrt{n}\right)^{2(2 k-1)}\right\}$ exists, and since $\varepsilon$ is as small as we need, we then obtain the desired result which completes Proposition 2.

\section{An analogue to a Markov process with discrete space}

We consider a Markov process $\left(X_{t}\right)_{t \in[0,+\infty)}$ on $\mathbb{Z}$ with $X_{0}=0$. The infinitesimal generator is defined by

$$
L_{\omega} f(k)=c\left(T^{k-1} \omega\right) f(k-1)+c\left(T^{k} \omega\right) f(k+1)-\bar{c}\left(T^{k} \omega\right) f(k) .
$$

We will now establish a CLT for a Markov process $\left(X_{t}\right)_{t \in[0,+\infty)}$ without the use of a martingale. 
Theorem 3. For almost all environments $\omega$,

$$
\frac{X_{t}}{\sqrt{t}} \stackrel{\mathrm{D}}{\rightarrow} \mathcal{N}\left(0,2\left[\int \frac{1}{c} \mathrm{~d} \mu\right]^{-1}\right) \text { as } t \rightarrow+\infty .
$$

The limiting distribution being a degenerate normal distribution if the integral is $+\infty$.

This problem was also considered by Kawazu and Kesten, [4]. They did not use the method of martingale but instead used a time change of a Brownian motion.

To prove Theorem 3 it is sufficient to show that for almost all environments $\omega$

$$
\lim _{t \rightarrow+\infty} \mathbb{E}_{\omega}\left\{\left(\frac{X_{t}}{\sqrt{t}}\right)^{\ell}\right\}=0 \quad \text { if } \ell=2 k-1, \quad \lim _{t \rightarrow+\infty} \mathbb{E}_{\omega}\left\{\left(\frac{X_{t}}{\sqrt{t}}\right)^{\ell}\right\}=\frac{(2 k) !}{k ! 2^{k}} \sigma^{\ell} \quad \text { if } \ell=2 k
$$

for each $\ell=1,2,3, \ldots$ In this case $\sigma^{2}=2\left[\int(1 / c) \mathrm{d} \mu\right]^{-1}$.

Lemma 5. Given a function $\psi: \mathbb{Z} \rightarrow \mathbb{R}$ there exists an unique function $\phi: \mathbb{Z} \rightarrow \mathbb{R}$ such that

$$
L_{\omega} \phi=\psi, \quad \phi(1)=a, \quad \phi(-1)=b .
$$

Proof. By a similar argument as in Lemma 2 we can show that (12) has a unique solution $\phi$ such that

- $\phi(1)=a$ and $\phi(-1)=b$;

- $\phi(0)$ satisfies $c\left(T^{-1} \omega\right) \phi(-1)+c(\omega) \phi(1)-\bar{c}(\omega) \phi(0)=\psi(0)$;

- $\phi(m)=\phi(1)+[\phi(1)-\phi(0)] \sum_{\ell=1}^{m-1} \frac{c(\omega)}{c\left(T^{\ell} \omega\right)}+\sum_{\ell=1}^{m-1} \frac{1}{c\left(T^{\ell} \omega\right)} \sum_{s=1}^{\ell} \psi(s)$ if $m \geq 2$;

- $\phi(m)=\phi(-1)+[\phi(-1)-\phi(0)] \sum_{\ell=2}^{-m} \frac{c\left(T^{-1} \omega\right)}{c\left(T^{-\ell} \omega\right)}+\sum_{\ell=2}^{-m} \frac{1}{c\left(T^{-\ell} \omega\right)} \sum_{s=1}^{\ell-1} \psi(-s)$ if $m \leq-2$.

Proposition 3. For almost all environments $\omega$, we have

$$
\lim _{t \rightarrow+\infty} \mathbb{E}_{\omega}\left\{\left(\frac{X_{t}}{\sqrt{t}}\right)^{2 k}\right\}=\frac{(2 k) !}{k ! 2^{k}} \sigma^{2 k} \text { for each } k \geq 1 .
$$

This limit is null if $c^{-1}$ is not integrable.

Proof. Fixing $\omega \in \Omega$, we consider a sequence of functions $f_{k} \geq 0$, defined on $\mathbb{Z}$, such that

$$
L_{\omega} f_{k} \equiv f_{k-1} \quad \text { for } k \geq 1, \quad f_{0} \equiv 1, \quad f_{k}(0)=0 \quad \text { for } k \geq 1 .
$$

By Lemma 5 we determine a function $f_{1}$ which satisfies $f_{1}(-1)=1 / c\left(T^{-1} \omega\right)$ and $f_{1}(1)=0$

$$
f_{1}(m)= \begin{cases}\sum_{\ell=1}^{m-1} \frac{\ell}{c\left(T^{\ell} \omega\right)} & \text { if } m \geq 2, \\ 0 & \text { if } m=0,1 \\ \sum_{\ell=1}^{-m} \frac{\ell}{c\left(T^{-\ell} \omega\right)} & \text { if } m \leq-1\end{cases}
$$


and for $k \geq 2$ a function $f_{k}$ which satisfies $f_{k}(1)=f_{k}(-1)=0$

$$
f_{k}(m)= \begin{cases}\sum_{\ell=1}^{m-1} \frac{1}{c\left(T^{\ell} \omega\right)} \sum_{s=1}^{\ell} f_{k-1}(s) & \text { if } m \geq 2 \\ 0 & \text { if } m=-1,0,1, \\ \sum_{\ell=2}^{-m} \frac{1}{c\left(T^{-\ell} \omega\right)} \sum_{s=1}^{\ell-1} f_{k-1}(-s) & \text { if } m \leq-2 .\end{cases}
$$

Then $L_{\omega} f_{k}(m)=f_{k-1}(m)$ for any integer $m$ and for $k \geq 1$. Replace $m$ by $X_{t}$ to obtain

$$
L_{\omega} f_{k}\left(X_{t}\right)=f_{k-1}\left(X_{t}\right) \text { for any } t \geq 0
$$

Hence we can show that for each $k \geq 1$

$$
\mathbb{E}_{\omega}\left\{f_{k}\left(X_{t}\right)\right\}=\frac{t^{k}}{k !} \quad \text { for any } t \geq 0 .
$$

Indeed, if $h_{1}(t)=\mathbb{E}_{\omega}\left\{f_{1}\left(X_{t}\right)\right\}$ then

$$
\begin{aligned}
\mathbb{E}_{\omega}\left\{L_{\omega} f_{1}\left(X_{t}\right)\right\} & =\lim _{s \rightarrow 0} \mathbb{E}_{\omega}\left\{\frac{\mathbb{E}_{\omega}\left\{f_{1}\left(X_{t+s}\right) / X_{t}\right\}-f_{1}\left(X_{t}\right)}{s}\right\} \\
& =\lim _{s \rightarrow 0} \mathbb{E}_{\omega}\left\{\frac{f_{1}\left(X_{t+s}\right)-f_{1}\left(X_{t}\right)}{s}\right\} \\
& =\lim _{s \rightarrow 0} \frac{h_{1}(t+s)-h_{1}(t)}{s}=h_{1}^{\prime}(t) .
\end{aligned}
$$

Since $\mathbb{E}_{\omega}\left\{L_{\omega} f_{1}\left(X_{t}\right)\right\}=1$ implies $h_{1}(t)=t+\beta$ for $t \geq 0$. And $h_{1}(0)=\mathbb{E}_{\omega}\left\{f_{1}\left(X_{0}\right)\right\}=0$ implies $\beta=0$, hence $h_{1}(t)=\mathbb{E}_{\omega}\left\{f_{1}\left(X_{t}\right)\right\}=t$. By induction on $k$ we obtain the proof of (13).

The expansion is similar to Proposition 1 in Section 2. The proof of Proposition 3 is complete.

Proposition 4. For almost all environments $\omega$, we have

$$
\lim _{t \rightarrow+\infty} \mathbb{E}_{\omega}\left\{\left(\frac{X_{t}}{\sqrt{t}}\right)^{(2 k-1)}\right\}=0 \text { for each } k \geq 1 .
$$

Proof. Fixing $\omega \in \Omega$ we consider a sequence of functions $g_{k}$, defined on $\mathbb{Z}$, such that

$$
L_{\omega} g_{k} \equiv g_{k-1} \quad \text { for } k \geq 1, \quad g_{0} \equiv 0, \quad g_{k}(0)=0 \quad \text { for } k \geq 1 .
$$

By Lemma 5 we can determine a function $g_{1}$ which satisfies $g_{1}(-1)=-1 / c\left(T^{-1} \omega\right)$ and $g_{1}(1)=1 / c(\omega)$

$$
g_{1}(m)= \begin{cases}\sum_{\ell=0}^{m-1} \frac{1}{c\left(T^{\ell} \omega\right)} & \text { if } m \geq 1 \\ 0 & \text { if } m=0 \\ -\sum_{\ell=1}^{-m} \frac{1}{c\left(T^{-\ell} \omega\right)} & \text { if } m \leq-1\end{cases}
$$


and for $k \geq 2$ a function $g_{k}$ which satisfies $g_{k}(1)=g_{k}(-1)=0$

$$
g_{k}(m)= \begin{cases}\sum_{\ell=1}^{m-1} \frac{1}{c\left(T^{\ell} \omega\right)} \sum_{s=1}^{\ell} g_{k-1}(s) & \text { if } m \geq 2, \\ 0 & \text { if } m=-1,0,1, \\ \sum_{\ell=2}^{-m} \frac{1}{c\left(T^{-\ell} \omega\right)} \sum_{s=1}^{\ell-1} g_{k-1}(-s) & \text { if } m \leq-2 .\end{cases}
$$

The expansion is similar to that in Proposition 2 in Section 2. The proof of Proposition 4 is thus complete.

Remark 1. We introduce the sequence of transition times, $\left(\tau_{n}\right)_{n \in \mathbb{N}}$, that are the times when $X_{t}$ jumps i.e. $\tau_{0}=0$ and

$$
\tau_{n}=\inf \left\{t \geq \tau_{n-1}: X_{t} \neq X_{\tau_{n-1}}\right\}
$$

The times between transition times $\tau_{n+1}-\tau_{n}$ are called waiting times. It is well known that the waiting times have an exponential distribution with the parameter $\bar{c}\left(T^{X_{\tau_{n}}} \omega\right)$ that depends only on the position of $X_{t}$ at time $\tau_{n}$. In this case $\tau_{n} \rightarrow \infty$ as $n \rightarrow \infty$.

The embedded process is given by $Y_{0}=X_{0}=0$ and

$$
Y_{n}=X_{\tau_{n}} .
$$

Then $\left(Y_{n}\right)_{n \geq 0}$ is also the random walk with transition probabilities as in the previous section. Hence we can deduce that $\left(X_{\tau_{n}}\right)_{n \geq 0}$ satisfies Theorem 2 .

\section{Diffusion in a stationary random environment}

Let $(\Omega, \mathcal{A}, \mu)$ be a probability space equipped with a flow $\left(T_{x}\right)_{x \in \mathbb{R}}$ that is ergodic and preserves the measure $\mu$. We consider two random variables $a, b>0$ such that the functions $x \mapsto a\left(T_{x} \omega\right)$ and $x \mapsto b\left(T_{x} \omega\right)$ are continuous. Now we look at where $\omega$ is fixed. The process of the infinitesimal generator is defined by

$$
L_{\omega} f(x)=\frac{1}{2 a\left(T_{x} \omega\right)} \frac{\mathrm{d}}{\mathrm{d} x}\left(b\left(T_{x} \omega\right) \frac{\mathrm{d} f}{\mathrm{~d} x}\right)
$$

with the initial condition $X_{0}=0$.

This describes the problem associated to the stochastic differential equation (SDE)

$$
\mathrm{d} X_{t}=\sigma_{\omega}\left(X_{t}\right) \mathrm{d} B_{t}+\mu_{\omega}\left(X_{t}\right) \mathrm{d} t,
$$

where $\left(B_{t}\right)_{t \geq 0}$ is a Brownian motion, the coefficient of diffusion $\sigma_{\omega}^{2}(x)=b\left(T_{x} \omega\right) / a\left(T_{x} \omega\right)$ and the drift $\mu_{\omega}(x)=\left(2 a\left(T_{x} \omega\right)\right)^{-1}(\mathrm{~d} / \mathrm{d} x)\left(b\left(T_{x} \omega\right)\right)$.

Theorem 4. Suppose that, almost anywhere $\omega \in \Omega$, the functions $\sigma_{\omega}^{2}(x)$ and $\mu_{\omega}(x)$ are locally Lipschitz. Then, for almost all $\omega \in \Omega$, the solution $\left(X_{t}\right)_{t \geq 0}$ of SDE (14) satisfies

$$
\frac{X_{t}}{\sqrt{t}} \stackrel{\mathrm{D}}{\rightarrow} \mathcal{N}\left(0,\left[\int_{\Omega} a \mathrm{~d} \mu \int_{\Omega} \frac{1}{b} \mathrm{~d} \mu\right]^{-1}\right) \text { as } t \rightarrow+\infty .
$$

The limiting distribution is a degenerate normal distribution if at least one of the integrals is $+\infty$. 
Papanicolaou and Varadhan [7] established the CLT for the elliptic case in dimension $d \geq 1$.

As in Theorem 2 we do not use any martingale to prove Theorem 4 . We begin with a continuous version of Lemma 1.

Lemma 6. Let $u(x)$ and $v(x)$ be two positive continuous functions and $\alpha$ a nonnegative integer. Assume that $\lim _{y \rightarrow+\infty}(1 / y) \int_{0}^{y} u(x) \mathrm{d} x=\bar{u}>0$ and $\lim _{x \rightarrow+\infty} v(x)=\bar{v}>0$. If both of $\bar{u}$ and $\bar{v}$ are finite then

$$
\lim _{y \rightarrow+\infty} \frac{1}{y^{\alpha+1}} \int_{0}^{y} x^{\alpha} u(x) v(x) \mathrm{d} x=\frac{\bar{u} \bar{v}}{\alpha+1}
$$

else if at least one of $\bar{u}$ and $\bar{v}$ is infinite then the limit in (15) is $+\infty$.

The proof of Lemma 6 is left to the reader.

Now we are going to show that for almost all environments $\omega$

$$
\lim _{t \rightarrow+\infty} \mathbb{E}_{\omega}\left\{\left(\frac{X_{t}}{\sqrt{t}}\right)^{\ell}\right\}= \begin{cases}0 & \text { if } \ell=2 k-1, \\ \frac{(2 k) !}{k ! 2^{k}} \sigma^{\ell} & \text { if } \ell=2 k\end{cases}
$$

for each $\ell=1,2,3, \ldots$ In this case $\sigma^{2}=\left[\int_{\Omega} a \mathrm{~d} \mu \int_{\Omega}(1 / b) \mathrm{d} \mu\right]^{-1}$.

Proposition 5. For almost all environments $\omega$, we have

$$
\lim _{t \rightarrow+\infty} \mathbb{E}_{\omega}\left\{\left(\frac{X_{t}}{\sqrt{t}}\right)^{2 k}\right\}=\frac{(2 k) !}{k ! 2^{k}} \sigma^{2 k}
$$

for each $k \geq 1$. This limit is null if at least one of a and $1 / b$ is not integrable.

This is the generalization of Theorem 4.1 in [3].

Proof. Fixing $\omega \in \Omega$ we consider a sequence of functions $f_{k}$, defined on $\mathbb{R}$, such that

$$
L_{\omega} f_{k} \equiv f_{k-1} \quad \text { for } k \geq 1, \quad f_{0} \equiv 1, \quad f_{k}(0)=0 \quad \text { for } k \geq 1 .
$$

For example, we can take

$$
f_{1}(x)= \begin{cases}\int_{v=0}^{x} \frac{1}{b\left(T_{v} \omega\right)} \int_{u=0}^{v} 2 a\left(T_{u} \omega\right) \mathrm{d} u \mathrm{~d} v & \text { if } x \geq 0 \\ \int_{v=x}^{0} \frac{1}{b\left(T_{v} \omega\right)} \int_{u=v}^{0} 2 a\left(T_{u} \omega\right) \mathrm{d} u \mathrm{~d} v & \text { if } x<0\end{cases}
$$

and for $k \geq 2$

$$
f_{k}(x)= \begin{cases}\int_{v=0}^{x} \frac{1}{b\left(T_{v} \omega\right)} \int_{u=0}^{v} 2 a\left(T_{u} \omega\right) f_{k-1}(u) \mathrm{d} u \mathrm{~d} v & \text { if } x \geq 0, \\ \int_{v=x}^{0} \frac{1}{b\left(T_{v} \omega\right)} \int_{u=v}^{0} 2 a\left(T_{u} \omega\right) f_{k-1}(u) \mathrm{d} u \mathrm{~d} v & \text { if } x<0 .\end{cases}
$$

Lemma 7. For each $k \geq 1$, we have

$$
\lim _{x \rightarrow \pm \infty} \frac{f_{k}(x)}{x^{2 k}}=\frac{2^{k}}{(2 k) !} \sigma^{-2 k} .
$$

This limit is $+\infty$ if at least one of a and $1 / b$ is not integrable. It is denoted by $\ell_{k}$.

The proof of Lemma 7 is left to the reader as a continuous version of Lemma 3. 
Moreover, by the hypothesis of the theorem, function $f_{k} \in \mathcal{C}^{2}$ for each $k \geq 1$, the process $Y_{t}^{(k)}$, defined by $Y_{t}^{(k)}=f_{k}\left(X_{t}\right)$, satisfies SDE (14) by using Itô's lemma. We recall that

$$
\mathrm{d} f_{k}\left(X_{t}\right)=f_{k}^{\prime}\left(X_{t}\right) \sigma_{\omega}\left(X_{t}\right) \mathrm{d} B_{t}+\left[f_{k}^{\prime}\left(X_{t}\right) \mu_{\omega}\left(X_{t}\right)+\frac{1}{2} f_{k}^{\prime \prime}\left(X_{t}\right) \sigma_{\omega}^{2}\left(X_{t}\right)\right] \mathrm{d} t .
$$

By calculating, $\mathrm{d} Y_{t}^{(k)}=c_{\omega}\left(X_{t}\right) \mathrm{d} B_{t}+f_{k-1}\left(X_{t}\right) \mathrm{d} t$ for each $k \geq 1$ where $f_{0} \equiv 1$. Hence, it follows that

$$
\mathbb{E}_{\omega}\left\{f_{k}\left(X_{t}\right)\right\}=\frac{t^{k}}{k !} \quad \text { for each } k \geq 1 .
$$

Combining Lemma 7 and (16) we obtain

$$
\left|\mathbb{E}_{\lambda, \omega}\left\{\left(\frac{X_{t}^{2}}{t}\right)^{k}\right\}-\frac{1}{k ! \ell_{k}}\right|<\varepsilon
$$

for any $\varepsilon>0$ and for $t$ large enough. And hence the proof of Proposition 5 is complete.

Proposition 6. For almost all environments $\omega$, we have

$$
\lim _{t \rightarrow+\infty} \mathbb{E}_{\omega}\left\{\left(\frac{X_{t}}{\sqrt{t}}\right)^{(2 k-1)}\right\}=0 \text { for each } k \geq 1 .
$$

Proof. Fixing $\omega \in \Omega$ we consider a sequence of functions $g_{k}$, defined on $\mathbb{R}$, such that

$$
L_{\omega} g_{k} \equiv g_{k-1} \quad \text { for } k \geq 1, \quad g_{0} \equiv 0, \quad g_{k}(0)=0, \quad \text { for } k \geq 1 .
$$

For instance, we can take

$$
g_{1}(x)=\int_{v=0}^{x} \frac{1}{b\left(T_{v} \omega\right)} \mathrm{d} v \quad \text { if } x \geq 0, \quad \text { and } \quad g_{1}(x)=-\int_{v=x}^{0} \frac{1}{b\left(T_{v} \omega\right)} \mathrm{d} v \quad \text { if } x<0
$$

and for $k \geq 2$

$$
g_{k}(x)= \begin{cases}\int_{v=0}^{x} \frac{1}{b\left(T_{v} \omega\right)} \int_{u=0}^{v} 2 a\left(T_{u} \omega\right) g_{k-1}(u) \mathrm{d} u \mathrm{~d} v & \text { if } x \geq 0, \\ \int_{v=x}^{0} \frac{1}{b\left(T_{v} \omega\right)} \int_{u=v}^{0} 2 a\left(T_{u} \omega\right) g_{k-1}(u) \mathrm{d} u \mathrm{~d} v & \text { if } x<0 .\end{cases}
$$

Lemma 8. For each $k \geq 1$, we have

$$
\lim _{x \rightarrow \pm \infty} \frac{g_{k}(x)}{x^{2 k-1}}=\frac{2^{k-1}}{(2 k-1) !} \sigma^{-2 k+2} \int \frac{1}{b} \mathrm{~d} \mu .
$$

This limit is $+\infty$ if at least one of a and $1 / b$ is not integrable.

The proof of Lemma 8 is left to the reader as a continuous version of Lemma 4 .

Moreover, by the hypothesis of the theorem, function $g_{k} \in \mathcal{C}^{2}$ for each $k \geq 1$, the process $Z_{t}^{(k)}$, defined by $Z_{t}^{(k)}=g_{k}\left(X_{t}\right)$, satisfies SDE (14) by using Itô's lemma. We recall that

$$
\mathrm{d} g_{k}\left(X_{t}\right)=g_{k}^{\prime}\left(X_{t}\right) \sigma_{\omega}\left(X_{t}\right) \mathrm{d} B_{t}+\left[g_{k}^{\prime}\left(X_{t}\right) \mu_{\omega}\left(X_{t}\right)+\frac{1}{2} g_{k}^{\prime \prime}\left(X_{t}\right) \sigma_{\omega}^{2}\left(X_{t}\right)\right] \mathrm{d} t .
$$

By calculating, $\mathrm{d} Z_{t}^{(k)}=d_{\omega}\left(X_{t}\right) \mathrm{d} B_{t}+g_{k-1}\left(X_{t}\right) \mathrm{d} t$ for each $k \geq 1$ and where $g_{0} \equiv 0$. Hence, it follows that

$$
\mathbb{E}_{\omega}\left\{g_{k}\left(X_{t}\right)\right\}=0 \quad \text { for each } k \geq 1 \text {. }
$$


Combining Lemma 8 and (17) we obtain

$$
\left|\mathbb{E}_{\lambda, \omega}\left\{\left(\frac{X_{t}}{\sqrt{t}}\right)^{2 k-1}\right\}\right|<\varepsilon
$$

for any $\varepsilon>0$ and for $t$ large enough. And hence the proof of Proposition 6 is complete.

Remark 2. In our model, there is no explosion in finite time under the assumptions concerning $\sigma_{\omega}^{2}(x)$ and $\mu_{\omega}(x)$ for almost all $\omega \in \Omega$. Indeed, we assume that there exists $\Gamma>0$ such that $\lim _{t \nearrow \Gamma} X_{t}=\infty$, where $X_{t}$ is a solution of SDE (14). Lemma 7 ensures that $f_{k}\left(X_{\gamma}\right)>\Gamma^{k} / k$ ! for some $\gamma \leq \Gamma$ and $k \geq 1$. This is a contradiction since we know from (16) that

$$
\mathbb{E}_{\omega}\left\{f_{k}\left(X_{\gamma}\right)\right\}=\frac{\gamma^{k}}{k !} \leq \frac{\Gamma^{k}}{k !} \quad \text { for each } k \geq 1 .
$$

\section{Acknowledgements}

This work was supported by the Israel Science Foundation (grant no. 1/12), as a postdoctoral fellowship at Ben Gurion University.

I am indebted to my $\mathrm{PhD}$ advisor, Jerome Depauw, for introducing this problem to me and for his very careful reading of an earlier version of this paper and his inspiring comments. I would like to thank Olivier Garet and the referee for their valuable suggestions.

\section{References}

[1] AliLi, S. (1999). Asymptotic behaviour for random walks in random environments. J. Appl. Prob. 36, 334-349.

[2] Billingsley, P. (1995). Probability and Measure, 3rd edn. John Wiley, New York.

[3] Depauw, J. and Derrien, J.-M. (2009). Variance limite d'une marche aléatoire réversible en milieu aléatoire sur Z. C. R. Math. Acad. Sci. Paris 347, 401-406.

[4] KawazU, K. And Kesten, H. (1984). On birth and death processes in symmetric random environment. J. Statist. Phys. 37, 561-576.

[5] KozLov, S. M. (1985). The averaging method and walks in inhomogeneous environments. Uspekhi Mat. Nauk 40, 61-120, 238.

[6] Mathieu, P. (2008). Quenched invariance principles for random walks with random conductances. J. Statist. Phys. 130, 1025-1046.

[7] Papanicolaou, G. C. and Varadhan, S. R. S. (1982). Diffusions with random coefficients. In Statistics and Probability: Essays in Honor of C. R. Rao, North-Holland, Amsterdam, pp. 547-552.

[8] Wiener, N. (1939). The ergodic theorem. Duke Math. J. 5, 1-18.

[9] Zeitouni, O. (2006). Random walks in random environments. J. Phys. A 39, R433-R464. 